la même heure, on obtenait seulement $75 \mathrm{gr}$,, et au bout de 7 jours, après quelques variations, la quantité journalière obtenue par une seule traite restait au voisinage de $60 \mathrm{gr}$., sans aucun phénomène de rétention, et se poursuivait ainsi pendant une vingtaine de jours, jusqu'à la cessation volontaire de l'observation (courbe E).

(A suivre.)

\title{
SUR L'INFLUENCE DE DIFFÉRENTS FACTEURS SUR LA TENDANCE A LA FERIMENTATION GAZEUSE PRÉCOCE DU FROMAGE
}

\author{
par \\ E. HAGLUND, E. SANDBERG et Chr. BARTHEL. \\ (Travail des Sections de laiterie et de bactériologie de l'Institut central \\ de Recherches agronomiques à Stockholm.)
}

Dans quelques travaux des années 1928-1930 de la Section de laiterie de l'Institut central de Recherehes agronomiques, nous avons traité la question de l'influence de la méthode de fabrication sur les transformations biochimiques et sur la consistance du fromage. De ces travaux, il ressort que la manière de saler et la quantité de sel ajoutée [1] ont une influence marquée sur le nombre bactérien et sur la rapidité de maturation du fromage, et, ensuite, que la consistance de celui-ci dépend en premier lieu du $p$ H à la fin de la fermentation lactique [2]. Un $p H$ élevé dans le fromage frais détermine une consistance souple et une pâte facile à couper, tandis que le fromage devient friable et de pâte courte à un $p$ H trop bas. Un $p H$ élevé est obtenu en premier lieu en employant un lait relativement pauvre en-bactéries lactiques. La fermentation lactique devient alors lente, la perte de chaux est peu considérable [3] et le fromage prend un $p H$ relativement élevé et une consistance souple.

La consistance des fromages à pâte dure a fortement attiré l'attention au cours de ces dernières années. Les efforts pour augmenter la consommation du fromage dans le pays a eu comme résultat que les exigences sont devenues beaucoup plus grandes en ce qui eoncerne sa consistance. Les laiteries cherchent donc, par des moyens techniques, à donner au fromage la consistance désirée. Cela amène une élévation du $p H$, et la question se pose alors de savoir si cette eirconstance augmente considérablement la tendance à la fermentation gazeuse précoce du fromage. Cette question fut rendue actuelle du fait qu'une laiterie demanda à la Section laitière de l'Institut de Recherches agronomiques d'essayer de trouver les causes de la fermentation gazeuse précoce que subit constamment le fromage de cette laiterie pendant et immédiatement après la mise sous presse. 
Puisque la fermentation apparut dans le fromage frais, contenant encore du lactose, on avait toute raison de supposer que la fermentation était causée par des bactéries appartenant au groupe coliaerogenes. De ces bactéries-là, ce sont surtout les aerogenes qui causent la fermentation gazeuse dans le fromage frais. Ceci fut prouvé, entre autres, par les recherches de Dorner [4], à la Station de bactériologie laitière de Liebefeld, à Berne. DORNER a trouvé que pendant la période où apparut à l'étable de Liebefeld la fermentation anormale, les aerogenes dominaient dans les excréments des vaches, tandis que, à partir du moment où la fermentation anormale du fromage avait cessé, les coli reprenaient leur rôle principal habituel. DoRNer dit aussi que la cause du trouble était due à un changement dans les espèces des bactéries fermentaires des excréments des vaches laitières, et que ses recherches ont confirmé ce qu'on a plusieurs fois remarqué, à savoir que ce sont surtout les aerogenes qui causent les fermentations anormales dans les fromages, tandis que les coli sont relativement très peu dangereux à ce point de vue.

Pour constater si, pour la laiterie suédoise en question, le Bact. aerogenes était la cause directe de la fermentation anormale, nous avons demandé à la laiterie de nous envoyer une quantité de son lait pour en fabriquer des fromages d'expérience, sous contrôle technique et bactériologique. L'acidité du lait était normale, mais la masse de fromage ne laissait échapper le sérum qu'avec difficulté. Le lactose était difficilement fermenté et la pâte du fromage frais avait une acidité anormalement basse. Le fromage fermentait déjà sous presse, de sorte que la masse sortait partiellement des moules. A cause de la fermentation, le fromage avait une texture anormale et le goût était très "impur». L'examen bactériologique du lait et du fromage frais montrait une telle abondance des aerogenes, qu'on pouvait sans hésitation attribuer la fermentation anormale à la présence de ces bactéries.

On aurait voulu se servir de ce lait naturellement infecté pour des recherches sur l'influence des différents facteurs techniques sur la fermentation gazeuse précoce, mais cela ne pouvait être réalisé, car la laiterie en question avait déjà cessé de fabriquer du fromage, vu les difficultés indiquées, et le lait était employé dans une autre laiterie où on ne fabriquait que du beurre.

Nous avions pourtant déjà isolé, du premier lait envoyé par la laiterie, un certain nombre de souches d'aerogenes. Dans les expériences décrites ci-dessous, nous avons ensemencé le lait employé pour la fabrication des fromages avec une de ces cultures.

\section{MÉTHODES ET EXPÉRIENCES PRÉLIMINAIRES.}

Pour faire des expériences avec un lait infecté d'une façon 
artificielle, il s'agissait d'employer une méthode d'ensemencement permettant d'obtenir dans les fromages une fermentation.gazeuse tout aussi forte que celle causée par le lait spontanément infecté et d'employer un appareillage qui permît de mesurer l'intensité de la fermentation.

L'influence de la méthode d'ensemencement sur la tendance du fromage à fermenter anormalement fut démontrée par les expériences suivantes :

Dans la première expérience, le fromage fut fabriqué avec du lait non pasteurisé, que l'on ensemença avee une culture en lait de Bact. aerogenes. Le fromage ainsi obtenu se boursoufla déjà sous presse, mais la fermentation fut bien plus faible qu'avec le lait spontanément infecté. Pour obtenir un développement plus rapide des bactéries ensemencées, on laissa le lait à la température de $30^{\circ}$ pendant une heure après l'ensemencement, pour " mûrir ». Cette façon d'opérer n'amena cependant pas une intensité plus grande de la fermentation et l'examen bactériologique montra que les bactéries lactiques ordinaires prédominaient dans le lait et dans le fromage frais. Pour arrêter la croissance des bactéries lactiques, le lait fut pasteurisé dans le bae pendant 10 minutes à $60^{\circ}$, puis refroidi jusqu'à $38^{\circ}$ et ensemencé avec 1,5\% d'une culture en lait de Bact. aerogenes; ensuite, il fut laissé au repos pendant une heure pour la "maturation ». Au bout de ce temps, il fut amené à la température d'emprésurage $\left(30^{\circ}\right)$ et ensemencé avant l'emprésurage avec une petite quantité de levain lactique. On chauffa ensuite la masse de fromage à $44^{\circ}$ et on la travailla pendant 60 minutes. Le fromage ainsi obtenu devint très boursouflé déjà sous presse et pendant les jours qui suivirent; l'intensité de la fermentation gazeuse parut être tout aussi forte qu'avec le lait original spontanément infecté. Des essais répétés ont donné les mêmes résultats. Il était done possible de fabriquer, au moyen des cultures d'aerogenes, un fromage montrant régulièrement une fermentation gazeuse précoce.

On essaya ensuite de trouver une méthode pour déterminer l'intensité de la fermentation en mesurant les quantités de gaz dégagées. Comme il était très difficile de doser le gaz contenu dans la masse même du fromage, on se contenta de mesurer les proportions du gaz carbonique et de l'hydrogène dégagées par le fromage. Ces quantités devaient être dans un certain rapport avec l'intensité de la fermentation, à la condition que tous les fromages fussent de mêmes dimensions, et que les déterminations des gaz fussent exécutées dans des conditions identiques.

Pour recueillir le gaz carbonique et l'hydrogène, le fromage fut placé sous une cloche en verre, reposant sur un plateau en fer ayant 
une rainure remplie de mercure, dans laquelle plongeait le bord de la cloche. L'air aspiré, qui passait par la cloche, fut préalablement débarrassé de son acide carbonique et, ensuite, à la sortie, séché sur de l'acide sulfurique et du chlorure de calcium. L'acide carbonique dégagé par le fromage était absorbé dans une solution potassique, et le gaz hydrogène restant fut chauffé dans un petit four électrique sur de l'oxyde de cuivre et dosé à l'état d'eau retenue dans un tube avec le chlorure de calcium. Deux fromages identiques furent ainsi mis dans deux appareils semblables et on cherchà à régler l'arrivée de l'air de sorte que la rapidité du passage de l'air fût le même dans les deux systèmes.

Tout d'abord, quelques expériences préliminaires furent exécutées en vue de constater si les quantités de gaz dosées étaient réellement en rapport direct avec la fermentation gazeuse du fromage, ou bien si elles étaient influencées aussi par d'autres transformations dans celui-ci.

Dans ce but, on fabriqua deux fromages avec du lait non pasteurisé, mis en moule sans salage, et pressés pendant 24 heures. Les fromages furent ensuite placés dans les cloches en verre et on y laissa passer de l'air débarrassé de l'acide carbonique. On détermina toutes les 24 heures les quantités d'acide carbonique et d'hydrogène dégagées. Pendant les premières 48 heures, la quantité d'acide carbonique fut assez constante, d'environ $0 \mathrm{gr}$. 20, mais augmenta ensuite fortement, pour monter après une semaine approximativement jusqu'à 1 gr. dans les 24 heures pour chaque fromage. Le gaz hydrogène resta pourtant presque eonstant pendant toute la durée de l'expérience.

Les cloches en verre ne furent pas enlevées pendant toute cette expérience et, par conséquent, la surface des fromages ne put être soumise à aucun traitement. Aussi, on y put constater l'apparition des moisissures déjà le deuxième jour, et cette végétation crût rapidement. On en put conclure que le dégagement croissant d'acide carbonique était dû à la végétation des moisissures.

Pour le constater d'une façon définitive, les cloches furent enlevées, dans les expériences suivantes, pendant un temps permettant un nettoyage de la surface des fromages. On put le faire sans nuire à l'expérience, parce que le volume d'air contenu dans les cloches était assez insignifiant par rapport à la quantité qui y passait pendant 24 heures. Pour empêcher le développement des moisissures, les fromages furent mis chaque jour, après l'enlèvement des cloches, dans des moules et des linges nouveaux, bouillis d'avance. Les linges et les moules étaient aussi préalablement lavés dans une solution concentrée d'acide borique. Comme les fromages étaient tout frais et 
devaient rester sous presse dans les cloches, on appliqua un poids en plomb sur leur surface libre.

A l'aide de cette méthode, on exécuta une expérience destinée à démontrer d'une façon directe l'influence du développement des moisissures sur la quantité d'acide carbonique dégagée. Les fromages employés, qui pesaient chacun $2 \mathrm{~kg}$. 5 , étaient faits de lait non pasteurisé additionné de $1 \%$ d'un levain lactique immédiatement avant l'emprésurage. Le caillé fut chauffé à $44^{\circ}$ et travaillé ensuite pendant 55 minutes, avant d'être mis en moule. Il n'y eut aucune addition de sel. Après être restés sous presse pendant une heure, la plus grande partie du sérum ayant été expulsée, les fromages furent mis sous les cloches et chargés de poids en plomb. Durant les cinq premiers jours, les moules et les linges furent traités comme nous l'avons décrit plus haut, et les surfaces des fromages ne montrèrent aucune trace de moisissure. La quantité d'acide carbonique dégagée par chaque fromage dans les 24 beures s'éleva à 0 gr. 05-0 gr. 10. Les cinq jours suivants, on ne traita plus la surface des fromages, mais on se contenta de les tourner, en enlevant pour un instant la cloche. Déjà le deuxième jour, après ce changement dans le traitement des fromages, on constata l'apparition des moisissures, qui se développèrent rapidement, de sorte que la quantité d'acide carbonique monta après une semaine jusqu'à $1 \mathrm{gr}$. dans les 24 heures pour chaque fromage. Quand on nettoyait les fromages de nouveau et qu'on faisait bouillir et traitait à l'acide borique les linges et les moules, la quantité d'acide carbonique dégagée diminuait de nouveau jusqu'à environ le même ehiffre qu'au commencement de l'expérience. Des expériences répétées ont donné les mêmes résultats. Il était donc prouvé que la végétation des moisissures sur le linge et sur les moules peut produire de telles quantités d'acide carbonique, que les quantités dégagées par les fromages mêmes sont complètement masquées. Pour obtenir des résultats valables, il est donc nécessaire d'empêcher tout développement de moisissures en traitant journellement les linges et les moules de la façon indiquée.

Pour juger de l'exactitude de la méthode employée dans nos expériences, il fallait évidemment connaître aussi la grandeur des variations dans les quantités d'acide carbonique chez des fromages provenant d'un même emprésurage. Pour éclaircir cette question on fit trois expériences, dans lesquelles on fabriqua chaque fois deux fromages non salés du même caillé, de $2 \mathrm{~kg}$. 5 chacun. Dans les deux premières de ces expériences, on employa un lait normal, tandis qu'à la troisième le lait fut ensemencé aveo une culture de Bact. aerogenes avant l'emprésurage. Dans chaque expérience, la 
quantité d'acide carbonique dégagée fut déterminée pendant les quatre premiers jours après la fabrication des fromages.

Les résultats obtenus sont exposés au tableau 1.

TABLEAU 1.

AGIDE GARBONIQUE DÉGAGÉ DE FROMAGES FABRIQUÉS PARALLÈLEMENT.

\begin{tabular}{|c|c|c|c|c|}
\hline Jour & I & 2 & 3 & 4 \\
\hline Acide carbonique & gr. & gr. & gr. & gr. \\
\hline 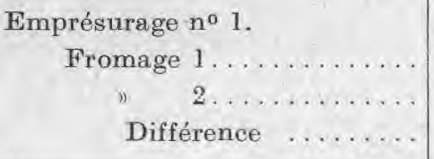 & $\begin{array}{r}0,12 \\
0,06 \\
+0,06\end{array}$ & $\begin{array}{r}0,11 \\
0,09 \\
+0,02\end{array}$ & $\begin{array}{r}0,12 \\
0,08 \\
+0,04\end{array}$ & $\begin{array}{r}0,12 \\
0,13 \\
-0,01\end{array}$ \\
\hline 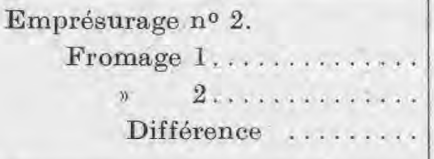 & $\begin{array}{r}0,18 \\
0,14 \\
+0,04\end{array}$ & $\begin{array}{r}0,18 \\
0,23 \\
-0,05\end{array}$ & $\begin{array}{r}0,19 \\
0,12 \\
+0,07\end{array}$ & $\begin{array}{r}0,: 4 \\
0,13 \\
+0,11\end{array}$ \\
\hline $\begin{array}{r}\text { Emprésurage } n^{0} 3 . \\
\text { Fromage } \begin{array}{r}1 \ldots \ldots \ldots \ldots \\
2 \ldots \ldots \ldots \ldots\end{array} \\
\text { Différence } \ldots \ldots \ldots \ldots\end{array}$ & $\begin{array}{r}0,49 \\
0,56 \\
+0,07\end{array}$ & $\begin{array}{r}0,52 \\
0,73 \\
-0,21\end{array}$ & $\begin{array}{r}0,68 \\
0,78 \\
-0,10\end{array}$ & $\begin{array}{r}0,16 \\
0,22 \\
-0,06\end{array}$ \\
\hline
\end{tabular}

Ce tableau montre que les fromages fabriqués parallèlement n'ont pas donné exactement la même quantité d'acide carbonique. La différence la plus grande se trouve chez les fromages provenant de l'emprésurage $n^{\circ} 3$. Cette différence de 0 gr. 21 représente environ la même quantité qui, à juger des emprésurages $n^{0 s} 1$ et 2 , est dégagée par un fromage normal. En général, les différences sont pourtant beaucoup moins grandes, et elles sont environ du même ordre de grandeur chaque jour. La différence la plus grande permet pourtant de constater que les fromages de l'emprésurage $\mathrm{n}^{0} \mathbf{3}$, malgré leur fermentation relativement faible, dégageaient une quantité bien plus grande d'acide carbonique que le font les fromages des deux premières séries. Il résulte done de ces expériences que la méthode employée pour juger l'intensité de la fermentation est utilisable.

Pour les expériences relatées, on s'est contenté de déterminer les quantités d'acide carbonique dégagées. Nos appareils étaient pourtant construits en vue de permettre aussi le dosage du gaz hydrogène dégagé.

Les fromages normaux, sans fermentation gazeuse précoce, ne produisirent que de très petites quantités d'hydrogène, de même 
que les fromages montrant le développement des moisissures, dont nous avons parlé au commencement. Il était donc intéressant de constater si les fromages qui fermentent dégageaient des quantités de gaz hydrogène assez considérables pour servir, elles aussi, à mesurer l'intensité de la fermentation. Il nous sembla aussi intéressant de savoir si les quantités des gaz carbonique et hydrogène étaient soumises à des changements réguliers pendant la marche de la fermentation. Nous avons suivi aussi la diminution du lactose et les changements du $p H$ du fromage qui en résultaient, en vue de constater si une durée d'expérience de 4 jours était suffisante.

Pour éelaircir ces questions, on exécuta des expériences avec des fromages normaux et fermentants. La totalité du lait employé pour une telle expérience fut pasteurisé à $63^{\circ}$ pendant 10 minutes et partagée entre deux bacs. Dans l'un, le lait fut refroidi jusqu'à la température de l'emprésurage $\left(30^{\circ}\right)$ et l'on ajouta la présure. Dans l'autre bac, le lait fut ramené à $38^{\circ}$, additionné de $1,5 \%$ d'une culture en lait de Bact. aerogenes et laissé à une "maturation" pendant une heure. Au bout de ce temps, ce lait fut à son tour refroidi à $30^{\circ}$ et emprésuré. Le découpage du caillé et le traitement de la masse furent ensuite exécutés exactement de la même manière dans les deux bacs. Le chauffage du caillé se fit à $44^{\circ}$ et le brassage final dura 50 minutes. Ce dernier travail demanda pourtant un temps un peu plus long pour le lait ensemencé avec le Bact. aerogenes.

Du lait de chaque bac, on fabriqua deux fromages non salés d'un poids de $2 \mathrm{~kg}$. 5 chaeun, L'un des fromages fut mis sous presse de façon ordinaire et, ensuite, laissé dans la saumure, tandis que l'autre, après une heure sous presse, était placé sous la eloche en verre de notre appareil, le poids en plomb étant posé sur sa surface supérieure. Une fois par jour, en même temps qu'on faisait les dosages des quantités de gaz d'acide earbonique et d'hydrogène, les cloches étaient enlevées et les fromages retournés et pourvus de nouveaux moules et linges, préalablement bouillis et lavés à l'acide borique. Par la même occasion, on prenait aussi des échantillons pour la détermination du lactose et du $p$ H. Les fromages qui avaient été pressés de façon ordinaire et salés ensuite dans la saumure, furent laissés à la maturation pendant six semaines et jugés ensuite au point de vue de la texture et du goût.

Le tableau 2 montre les résultats obtenus avec trois emprésurages parallèles, exécutés de la façon décrite plus haut.

On trouve, dans ce tableau, que pour le fromage fermenté les quantités d'acide carbonique dégagées furent bien plus grandes que pour le fromage normal. Pour le premier, on obtint jusqu'à 2-3 gr. dans les 24 heures, tandis que le fromage normal ne dégagea au plus que $0 \mathrm{gr}$. 30. Il y eut encore cette différence, que pour le fromage 
fermenté les quantités de gaz montèrent à un maximum pendant le deuxième et le troisième jour, pour diminuer ensuite très vite.

TABLEAU 2.

LE DÉGAGEMENT DE GAZ, ETG., DANS DES FROMAGES FERMENTÉS ET NORMAUX.

\begin{tabular}{|c|c|c|c|c|c|c|c|c|c|}
\hline \multirow[b]{2}{*}{ Emprésurage } & \multirow[b]{2}{*}{ Jour } & \multicolumn{4}{|c|}{ Fromage fermenté } & \multicolumn{4}{|c|}{ Fromage normal } \\
\hline & & $\begin{array}{l}\mathrm{CO}^{2} \\
\text { gr. }\end{array}$ & $\begin{array}{c}\text { Eau, par } \\
\text { eombustion } \\
\text { de } \mathrm{H}^{2} \text { en gr. }\end{array}$ & $\begin{array}{c}\text { Lactose } \\
\%\end{array}$ & $p \mathrm{H}$ & $\begin{array}{l}\mathrm{CO}^{2} \\
\mathrm{gr} .\end{array}$ & $\begin{array}{c}\text { Eau, par } \\
\text { combustion } \\
\text { de } H^{2} \text { en gr. }\end{array}$ & $\begin{array}{c}\text { Laetose } \\
\%\end{array}$ & $p \mathrm{H}$ \\
\hline \multirow{4}{*}{ No 1} & 1 & 1,62 & 0,15 & 0,67 & - & 0,16 & 0,01 & 0,21 & - \\
\hline & 2 & 1,87 & 0,16 & 0,25 & 5,94 & 0,08 & 0,00 & 0,16 & 4,93 \\
\hline & 3 & 2,37 & 0,29 & 0,07 & 5,81 & - & - & 0,00 & 4,91 \\
\hline & 4 & 0,48 & 0,02 & 0,00 & 一 & 0,29 & 0,01 & 0,00 & - \\
\hline \multirow{4}{*}{ No $\angle$} & 1 & 0,40 & 0,02 & 0,95 & 5,96 & 0,06 & 0,01 & 0,19 & 4,94 \\
\hline & 2 & 2,13 & 0,17 & 0,18 & 5,23 & 0,15 & 0,00 & 0,06 & 4,93 \\
\hline & 3 & 0,83 & 0,07 & 0,00 & 5,35 & 0,13 & 0,00 & 0,00 & 4,87 \\
\hline & 4 & 1,01 & 0,05 & 0,00 & - & 0,24 & 0,00 & 0,00 & 一 \\
\hline \multirow{4}{*}{ No 3} & 1 & 2,05 & 0,26 & 1,04 & 6,28 & 0,05 & 0,02 & 0,16 & 4,87 \\
\hline & 2 & 3,18 & 0,60 & 0,22 & 6,14 & 0,06 & 0,01 & 0,10 & 4,87 \\
\hline & 3 & 1,07 & 0,08 & 0,00 & 6,00 & 0,30 & 0,01 & 0,00 & 4,87 \\
\hline & 4 & 0,17 & 0,03 & 0,00 & - & 0,07 & 一 & 0,00 & - \\
\hline
\end{tabular}

Le fromage normal, par contre, dégagea environ la même quantité de gaz par jour pendant tout le temps de l'expérience.

Le gaz hydrogène fut dosé à l'état d'eau. Pour les fromages fermentés, les chiffres du tableau 2 montrent que la quantité d'hydrogène dégagée dans les 24 heures augmente et diminue généralement parallèlement avec l'acide carbonique. Puisque le fromage normal, selon le tableau 2, ne dégage que de très petites quantités d'hydrogène, tandis que le fromage fermenté en forme des quantités considérables, il est évident que le gaz hydrogène pourra aussi servir pour mesurer l'intensité de la fermentation.

Des chiffres sur la teneur des fromages en lactose, on déduit que la fermentation de celui-ci se fait le plus rapidement dans le fromage normal, mais que même dans le fromage fermenté tout le lactose a disparu après trois jours. Cela prouve done que le temps employé pour ces expériences fut suffisamment long.

En ce qui concerne l'acidité réelle des fromages, nous remarquons que le fromage fermenté montre constamment un $p H$ plus élevé que le fromage normal. L'intensité de la fermentation anormale s'exprime 
en quelque sorte dans le $p \mathrm{H}$ obtenu pour le fromage frais. A en juger par les quantités des gaz dégagées, la fermentation la plus forte s'est manifestée dans l'emprésurage no 3 . Le fromage fermenté provenant de cet emprésurage a montré aussi, après la disparition du lactose, le $p \mathrm{H}$ le plus haut : 6,00 et au-dessus.

Les fromages pressés et salés de la façon ordinaire, provenant des mêmes emprésurages que ceux du tableau 2, furent examinés à l'âge de 6 semaines. Tous les fromages fermentés montrèrent une texture anormale et un goût amer et "impur", tandis que les fromages normaux avaient une bonne texture, peut-être un peu trop fermée, et un goût sans défaut.

Les expériences relatées jusqu'ici prouvent ainsi qu'il est possible, en mesurant les quantités d'acide carbonique et d'hydrogène dégagées pendant les 4 premiers jours après la fabrication, de se rendre compte de l'intensité d'une fermentation anormale dans le fromage frais, causée par des bactéries du groupe aerogenes.

\section{L'INFLUENCE DES DIFFÉRENTES MANIPULATIONS TECHNIQUES SUR L'INTENSITÉ DE LA FERMENTATION GAZEUSE.}

Dorner [5] a démontré que les conditions de développement dans le lait des bactéries fermentaires dépendent du nombre de ces bactéries par rapport au nombre des vraies bactéries lactiques et du pouvoir fermentatif et de la faculté de croissance des organismes fermentaires. DoRner fait aussi remarquer que le fait déjà connu, qu'on ne réussit pas toujours à transmettre par ensemencement un défaut bactériologique d'un lait à un autre lait, prouve que le lait même peut être plus ou moins favorable comme milieu de culture pour les différentes bactéries.

Pour la fabrication des fromages, on cherche quelquefois à empêcher le développement des bactéries nuisibles en ajoutant au lait du levain lactique. Cette opération a pour résultat en même temps d'augmenter le nombre des bactéries lactiques et d'accélérer la vitesse de la fermentation lactique, ce qui fait que le fromage obtient plus vite un degré plus haut d'acidité actuelle.

Nous avons fait quelques expériences pour démontrer l'influence des bactéries lactiques sur la fermentation anormale dans les fromages, eausée par les aerogenes. Le lait fut pasteurisé à $63^{\circ}$ pendant 10 minutes, puis refroidi à $38^{\circ}$ et ensemencé avec 1,5\% d'une eulture en lait de Bact. aerogenes. Il fut alors abandonné à une "maturation" pendant une heure, refroidi à la température d'emprésurage et partagé entre deux bacs. Dans l'un de ces bacs le lait fut, avant l'emprésurage, additionné d'une certaine quantité de levain lactique, tandis que le lait de l'autre bac était emprésuré tel quel. Le fromage fabriqué avec le lait additionné du levain lactique fut appelé "fromage d'expérience", alors que l'autre était le 
"fromage de contrôle". Ce dernier devait montrer si les aerogenes ensemencés s'étaient réellement développés et s'ils possédaient bien la faculté de provoquer le boursouflement. De ehaque bac, on obtint deux fromages, d'un poids de $2 \mathrm{~kg}$. 5 chacun. L'un de ces fromages fut employé pour la détermination des gaz dégagés pendant la fermentation, tandis que l'autre fut mis sous presse de la façon ordinaire, placé dans la saumure et laissé à la maturation pendant 6 semaines; ce dernier fut ensuite examiné au sujet de son degré de maturation et de sa qualité générale. Dans le tableau 3, on trouve exposés les résultats de trois emprésurages exécutés de la façon décrite ci-dessus.

TABLEAU 3.

INFLUENCE DE LA PASTEURISATION DU LAIT SUR LA FERMENTATION DU FROMAGE.

\begin{tabular}{|c|c|c|c|c|c|c|c|c|c|c|c|}
\hline \multirow[b]{2}{*}{$\begin{array}{l}\text { Empré- } \\
\text { surage }\end{array}$} & \multirow[b]{2}{*}{ Jour } & \multicolumn{5}{|c|}{ Fromage d'expérience } & \multicolumn{5}{|c|}{ Fromage de contrôle } \\
\hline & & $\begin{array}{c}\text { Levain } \\
\text { lactique } \\
\%\end{array}$ & $\begin{array}{l}\mathrm{CO}^{s} \\
\text { gr. }\end{array}$ & \begin{tabular}{|c|} 
Eau, par \\
combus- \\
tion de $\mathrm{H}^{\mathrm{2}}$ \\
gr.
\end{tabular} & $\begin{array}{c}\text { Lactose } \\
\%\end{array}$ & $p \mathrm{H}$ & $\begin{array}{c}\text { Levain } \\
\text { lactique } \\
\% \\
\end{array}$ & $\begin{array}{l}\mathrm{CO}^{2} \\
\text { gr. }\end{array}$ & \begin{tabular}{|c} 
Eau, par \\
combus- \\
tion de $\mathrm{H}^{2}$ \\
gr.
\end{tabular} & $\begin{array}{c}\text { Lactose } \\
\%\end{array}$ & $p \mathrm{H}$ \\
\hline No 1 & $\begin{array}{l}1 \\
2 \\
3 \\
4\end{array}$ & 1 & $\begin{array}{l}1,17 \\
0,54 \\
0,22 \\
0,33\end{array}$ & $\begin{array}{l}0,44 \\
0,18 \\
0,05 \\
0,03\end{array}$ & $\begin{array}{l}0,16 \\
0,09 \\
0,07 \\
0,00\end{array}$ & $\begin{array}{l}5,10 \\
5,01 \\
4,98 \\
4,98\end{array}$ & 0 & $\begin{array}{l}2,70 \\
3,46 \\
0,81 \\
0,10\end{array}$ & $\begin{array}{l}0,44 \\
0,53 \\
0,13 \\
0,01\end{array}$ & $\begin{array}{l}0,61 \\
0,25 \\
0,18 \\
0,00\end{array}$ & $\begin{array}{l}6,02 \\
5,94 \\
5,86 \\
5,86\end{array}$ \\
\hline No 2 & $\begin{array}{l}1 \\
2 \\
3 \\
4\end{array}$ & 2 & $\begin{array}{l}0,21 \\
0,22 \\
0,13 \\
0,22\end{array}$ & $\begin{array}{l}0,05 \\
0,03 \\
0,09 \\
0,09\end{array}$ & $\begin{array}{l}0,24 \\
0,17 \\
0,05 \\
0,00\end{array}$ & $\begin{array}{l}5,03 \\
5,00 \\
4,97 \\
4,97\end{array}$ & 0 & $\begin{array}{l}2,26 \\
3,05 \\
0,52 \\
0,51\end{array}$ & $\begin{array}{l}0,31 \\
0,49 \\
0,09 \\
0,03\end{array}$ & $\begin{array}{l}0,63 \\
0,20 \\
0,10 \\
0,00\end{array}$ & $\begin{array}{l}5,88 \\
5,77 \\
5,74 \\
5,74\end{array}$ \\
\hline No 3 & $\begin{array}{l}1 \\
2 \\
3 \\
4\end{array}$ & 3 & $\begin{array}{l}0,19 \\
0,23 \\
0,31 \\
0,37\end{array}$ & $\begin{array}{l}0,03 \\
0,02 \\
0,01 \\
0,01\end{array}$ & $\begin{array}{l}0,09 \\
0,07 \\
0,00 \\
0,00\end{array}$ & $\begin{array}{r}4,77 \\
4,72 \\
4,72 \\
4,72\end{array}$ & 0 & $\begin{array}{l}1,85 \\
1,11 \\
0,66 \\
0,55\end{array}$ & $\begin{array}{l}0,46 \\
0,16 \\
0,13 \\
0,10\end{array}$ & $\begin{array}{l}0,49 \\
0,46 \\
0,15 \\
0,00\end{array}$ & $\begin{array}{l}5,58 \\
5,33 \\
5,43 \\
5,32\end{array}$ \\
\hline
\end{tabular}

On voit, dans ce tableau, que le lait fut additionné de 1,2 et $3 \%$ respectivement de levain lactique, tandis que le fromage de contrôle fut fabriqué avec un lait sans une telle addition. A l'emprésurage $\mathrm{n}^{\circ} 1$, où l'on avait ajouté seulement $1 \%$ de levain lactique pour le fromage d'expérience, celui-ci dégagea des quantités considérables d'acide carbonique et d'hydrogène: $1 \mathrm{gr}$. $17 \mathrm{de} \mathrm{CO}^{2}$ et $0 \mathrm{gr}$. $44 \mathrm{~d}^{\prime} \mathrm{H}^{2} \mathrm{O}$. En augmentant la quantité de levain lactique ajoutée jusqu'à 
$2 \%$ (emprésurage no 2), les quantités des gaz dégagées par le fromage d'expérience furent beaucoup moins grandes. Le gaz hydrogène se dégagea pourtant toujours en quantités relativement considérables, car les quantités d'eau déterminées s'élevèrent à 0 gr. 03-0 gr. 09 par heure. Ce ne fut qu'après avoir ajouté $3 \%$ de levain lactique au lait d'emprésurage, que les quantités de gaz hydrogène provenant du fromage d'expérience furent comparables à celles dégagées par des fromages normaux selon le tableau 2.

Au fur et à mesure qu'on augmentait la quantité de levain lactique ajoutée, la vitesse de la fermentation du lactose dans les fromages augmentait également. Ainsi, dans les deux premiers emprésurages le lactose ne disparut des fromages d'expérience que le quatrième jour ; mais avee le dernier emprésurage, il disparut déjà au bout du troisième jour. La vitesse différente avee laquelle le lactose disparaissait a aussi eu son effet sur l'acidité actuelle des fromages d'expérience. Ainsi, le fromage d'expérience de l'emprésurage $\mathrm{n}^{\circ} 3$ a montré, après la disparition complète du lactose, un $p \mathrm{H}$ bien plus bas que les deux autres.

Tous les fromages de contrôle ont dégagé des quantités considérables de gaz carbonique et d'hydrogène. Cela prouve que les aerogenes ensemencés dans le lait s'étajent très bien développés, et qu'ils possédaient la faculté de produire le boursouflement du fromage. Le lactose disparaissait un peu plus lentement dans les fromages de contrôle que dans les fromages d'expérience, mais même dans les premiers, le lactose avait disparu à la fin du quatrième jour. Ainsi que pour les fromages fermentés du tableau 2, les fromages de contrôle du tableau 3 montrent des valeurs de $p H$ très élevées.

Les fromages de contrôle conservés sous des conditions pratiques ont tous montré une texture spongieuse, une consistance de cuir et un goût très amer et “impur », Les fromages d'expérience, conservés de la même manière, ont montré une texture normale, peut-être un peu trop fermée. La pâte était courte. Elle avait un défaut qui était en rapport direct avec la quantité de levain lactique ajoutée au lait d'emprésurage. Le goût des fromages d'expérience était un peu amer et " impur " pour les deux premiers emprésurages, tandis que le fromage provenant de l'emprésurage $n^{\circ} 3$, où le lait avait été additionné de $3 \%$ de levain lactique, avait un goût normal.

Ainsi, l'expérience relatée ci-dessus a démontré qu'il est possible de prévenir une fermentation anormale précoce dans le fromage, en ajoutant du levain lactique au lait d'emprésurage. Si l'on en ajoute trop, on obtient cependant une valeur basse du $p H$ et une consistance courte.

(A suivre.) 